\title{
Sugerencias para el manejo de pacientes de cirugía general durante la emergencia sanitaria por COVID-19
}

\section{Suggestions for the management of general surgery patients during the Covid-19 health emergency}

Correspondencia Yeray Trujillo Loli trujilloyeray@gmail.com

Recibido: 16/04/2020 Arbitrado por pares Aprobado: 22/07/2020

Citar como: Trujillo-Loli Y, CabreraPastor A, Castañeda Puicon Ll. Sugerencias para el manejo de pacientes de cirugía general durante la emergencia sanitaria por COVID-19. Acta Med Peru. 2020;37(3):382-9. doi: https://doi. org/10.35663/amp.2020.373.940

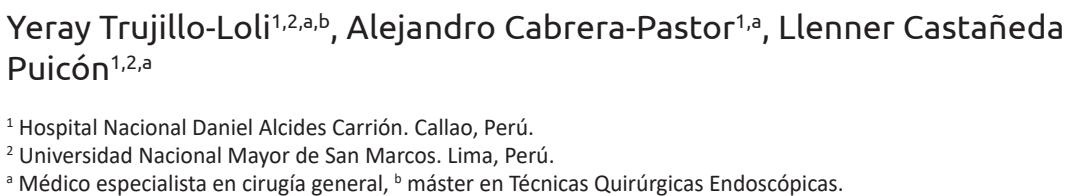

\section{RESUMEN}

El objetivo del presente artículo es brindar sugerencias, para el manejo pre, intra y posoperatorio, de pacientes de cirugía general, durante la emergencia sanitaria por COVID-19; y con ello, minimizar el contagio de cirujanos y disminuir la transmisión intrahospitalaria de la enfermedad. Se realizó una revisión de la literatura a través de PubMed y se consultaron las páginas web de sociedades científicas internacionales, así como, fuentes del Ministerio de Salud del Perú. Se formularon sugerencias para el manejo de estos pacientes, basadas en la experiencia de países donde se tiene un mayor número de casos de COVID-19. Se resalta la importancia del uso adecuado de los equipos de protección personal (EPPS); y la factibilidad de realizar un abordaje abierto o laparoscópico en cirugías de emergencia con los cuidados adecuados para evitar contagios en el paciente y personal de salud. Sin embargo, se debe considerar que las recomendaciones pueden cambiar con el tiempo al generarse nuevos conocimientos.

Palabras clave: Cirugía general; Laparoscopía; COVID-19 (fuente: DeCS-BIREME).

\begin{abstract}
The objective of this paper is to present suggestions for the preoperative, intraoperative, and postoperative management of general surgery patients during the COVID-19 health emergency, aiming to minimize risks for surgeons and to reduce in-hospital transmission of SARS-CoV- 2 . We reviewed the literature using the PubMed database and we also reviewed websites from international scientific societies and the Peruvian Ministry of Health website. Suggestions for the management of surgical patients are proposed, based on the experience of countries with larger numbers of COVID-19 cases. We emphasize the importance of the adequate use of personal protective equipment (PPE), and the feasibility of using an open or laparoscopy approach in emergency surgical procedures, taking adequate care in order to avoid viral transmission for both the patient and healthcare personnel. Nonetheless, we must consider that recommendations may vary with time as new knowledge is acquired.
\end{abstract}

Keywords: General surgery; Laparoscopy; COVID-19 (source: MeSH NLM). 


\section{INTRODUCCIÓN}

En diciembre del año 2019, un tipo nuevo de neumonía por Coronavirus (SARS-CoV-2) surgió en Wuhan-China, que en adelante se denominó COVID-19 ${ }^{[1]}$. La enfermedad se extendió en los siguientes meses por todo el mundo y el 11 de marzo del 2020 fue reconocida como una pandemia por la Organización Mundial de la Salud (OMS). El COVID-19 llegó al Perú el 6 de marzo del 2020, y 10 días después, se decretó el estado de emergencia y cuarentena nacional debido a las consecuencias de esta enfermedad ${ }^{[2]}$.

Como sucedió en otras epidemias, la mayoría de países asumió la posición de restringir las intervenciones quirúrgicas y sólo atender casos de emergencia ${ }^{[3]}$. Adicional a ello, surge el problema del contagio al personal de salud; por ejemplo, en España las estadísticas indican que el $24,1 \%$ de contagiados pertenece al personal sanitario ${ }^{[4]}$, lo que tiene un especial impacto en esta situación, debido a que se pierden profesionales valiosos en la lucha contra la pandemia.

Otro problema que limita el desarrollo de intervenciones quirúrgicas en el medio de la pandemia por COVID-19 es las características de esta infección. Por ejemplo, el periodo de incubación de la enfermedad oscila entre 2 a 14 días; mientras que la mediana de tiempo desde los primeros síntomas hasta que presentan disnea e ingreso hospitalario es de 5 a 7 días; lo que abre la posibilidad de un buen grupo de pacientes que se encuentren asintomáticos, pero en periodo infeccioso ${ }^{[5,6]}$.

Además, se ha detectado la presencia de este virus en heces (29\%) y una escasa proporción en sangre $(1 \%)^{[7]}$ lo que constituye un problema para el cirujano en el acto quirúrgico por el riesgo de contagio. En otras situaciones similares, se han encontrado trabajos que identifican otros agentes como el virus de la hepatitis $B$ y virus del papiloma humano en el humo quirúrgico aerosolizado, tanto en cirugía abierta como en cirugía laparoscópica ${ }^{[8,9]}$; motivo por el cual, se recomienda que el equipo quirúrgico debe estar protegido de la aerosolización usando un Equipo de Protección Personal (EPP) con la mascarilla adecuada ${ }^{[10-12]}$ y un equipo de aspiración y filtros de humo quirúrgico en un sistema cerrado para evitar la fuga del mismo al quirófano ${ }^{[11]}$ y evitar un probable contagio por ésta vía. Aunque en estos casos, esta vía de transmisión aún no está demostrada, estas recomendaciones si son aplicables ante la prevención del contagio de COVID-19 en sala de operaciones.

Sin embargo, en este contexto si bien las medidas de precaución actuales disminuyen la probabilidad de contagio, no son suficientes para detener la aparición de COVID-19 en cirujanos. En el Hospital Universitario La Paz en Madrid-España, el $24,4 \%$ de cirujanos generales dieron resultado positivo a COVID-19, de los cuales sólo el $2 \%$ contrajo la enfermedad luego de tomar las medidas de precaución para prevenir el contagio ${ }^{[6]}$. Por ello, es importante que los establecimientos de salud de mediana y alta complejidad, con servicios quirúrgicos, establezcan sugerencias dentro de sus especialidades. Es importante resaltar que tanto la Organización Mundial de la Salud (OMS) y el Ministerio de Salud del Perú (MINSA), no han dado sugerencias para prevenir el contagio del personal de salud durante una cirugía.

Las medidas de prevención que se tomen ante situaciones de contacto con pacientes sospechosos y/o confirmados de infección por SARS-CoV-2, son fundamentales para disminuir la tasa de infección nosocomial. En algunos centros hospitalarios, la tasa de infección se encuentra alrededor del $43 \%$, sobre todo en el área quirúrgica ${ }^{[13]}$.

Por lo señalado anteriormente, es necesario establecer sugerencias perioperatorias, tomadas de las experiencias internacionales. Países como Estados Unidos, España, Italia y China, se vieron afectados con una gran cantidad de casos por COVID-19 antes que el Perú; por ello, la experiencia de estos países resulta importante ${ }^{[14]}$. En esta revisión, dividiremos el tipo de manejo según el momento en que se encuentre el paciente en la etapa quirúrgica, es decir, en el preoperatorio, intraoperatorio y postoperatorio.

\section{SUGERENCIAS EN EL PREOPERATORIO}

Todo paciente requiere exámenes de laboratorio de rutina ${ }^{[15]} ;$ sin embargo, frente a esta pandemia, son necesarios otros exámenes para complementar el estudio de pacientes sospechosos de COVID-19, como son la radiografía de tórax y una tomografía torácica, donde se puede visualizar infiltraciones y opacidades unilaterales o bilaterales, engrosamiento septal inter e intralobular intersticial, entre otros hallazgos ${ }^{[16]}$.

Las siguientes sugerencias permiten disminuir la tasa de infección intrahospitalaria y proporcionar atención quirúrgica oportuna al paciente ${ }^{[17,18] \text { : }}$

1. Todo paciente potencialmente quirúrgico y que se encuentre hemodinámicamente estable, sea un caso descartado, sospechoso o positivo, debe ser intervenido de emergencia por el equipo de cirugía, teniendo la mayor certeza en el diagnóstico establecido, valorando el manejo conservador/ médico en algunas patologías.

2. Realizar la cirugía si es que ésta, al no llevarla a cabo o falla en el manejo conservador, va a prolongar la estadía hospitalaria, probabilidad de reingreso al nosocomio o genera complicaciones posteriores.

3. Todo paciente sospechoso que vaya a ser intervenido de cirugía de emergencia se debe realizar una historia clínica adecuada, prueba serológica rápida para COVID-19, toma de radiografía de tórax y en caso que se someta a una tomografía abdominal, agregar una tomografía torácica ${ }^{[19]}$.

4. Considerar a todos los pacientes como sospechosos o confirmados.

5. El equipo quirúrgico requiere de EPP y el material quirúrgico necesario, como se detalla más adelante. 
6. Solo el personal que vaya a realizar la intubación, en caso sea necesario anestesia de tipo general, debe mantenerse en la sala, los demás deben esperar afuera de ésta hasta que se concluya dicho procedimiento.

7. Las recomendaciones internacionales indican que debe existir un espacio intermedio o antesala (Tabla 1) que cumpla las siguientes funciones:

a. Intercambio de materiales o implementos necesarios para la cirugía.

b. Zona de retiro de ropa quirúrgica complementaria.

Tabla 1. Manejo del EPP según área específica.

\begin{tabular}{ll}
\multicolumn{1}{c}{ Área } & \multicolumn{1}{c}{ Equipo a retirar } \\
Sala de operaciones & Bata \\
& Protector visual \\
& Un gorro \\
& Un par de botas \\
& Un par de guantes \\
& Mascarilla quirúrgica \\
& Overol \\
Antesala o afín & Un gorro \\
& Un par de botas \\
& Mascarilla N95 \\
& Lentes \\
& Un par de guantes. \\
\hline
\end{tabular}

\section{Bioseguridad del personal de salud}

En primer lugar, se tiene el uso del EPP estéril en casos de pacientes confirmados y sospechosos, que incluye ${ }^{[12,20]}$ :

a. Doble set de ropa quirúrgica.

b. Overol con capucha impermeable.

c. Bata o mandilón quirúrgico impermeable.

d. Mascarilla: Las mascarillas necesarias son las N95, FFP2 o FFP3 y sobre éstas se puede colocar una mascarilla quirúrgica, si se tiene intención de reutilizar las primeras; ya que existe alta penetración de partículas en las mascarillas simples.

e. Lentes: Se requiere un cobertor facial completo, lentes con pantalla parcial o gafas de montura integral para evitar salpicaduras, pero sobre todo la producción de aerosoles.

f. Pantalla de cobertura facial: Así como los lentes o gafas protectoras, las pantallas protegen solo a las salpicaduras, pero no de aerosolización, aun así, se pueden usar sobre las gafas.

g. Guantes: En el caso de cirugías éstos deben ser estériles y se recomienda el uso de doble guante.

h. Gorras: De preferencia descartables, uso de doble gorra. En mujeres es esencial que cubra el cabello, sujeto por un moño.

i. Botas: De preferencia descartables, uso de doble bota. De uso único para el procedimiento quirúrgico, verificar el sellado y uso de calzado impermeable que luego pase por procedimiento de desinfección.

j. Equipo estéril para la intervención quirúrgica.
Otro aspecto a considerar es el uso de espacios alternos y separados para el manejo de pacientes sospechosos/ confirmados, lo que requiere una sala de operaciones exclusiva para estos casos, que mantenga una presión negativa por debajo de -5 Pascal $(\mathrm{Pa})$ o con filtros tipo "High Efficiency Particulate Air" (HEPA), donde exista intercambio frecuente del aire ${ }^{[20,21]}$. Este tipo de quirófanos a presión negativa o con filtros tipo HEPA, deben utilizarse si están disponibles, de no contar con esta tecnología se pueden utilizar quirófanos a presión neutra (quirófano convencional), recordando no apagar el sistema de ventilación durante el tiempo que dure la epidemia, el cual debe estar alejado de los otros quirófanos. En el caso de quirófanos a presión negativa o con filtros tipo HEPA, si se cuenta con 10 recambios de aire por hora, se debe esperar entre cada cirugía 30 minutos, si se cuenta con dos recambios, se debe de esperar 138 minutos, y si el quirófano es a presión neutra (sin recambios) se debe esperar tres horas ${ }^{[22,23]}$

Finalmente, la OMS y el MINSA, describen una secuencia para colocarse y retirarse el EPP que está enfocada a la atención de un paciente sospechoso de COVID-19, pero no se orientan al acto quirúrgico donde también se debe garantizar la correcta asepsia y antisepsia para prevenir infecciones intrahospitalarias, por ello la secuencia de colocación y retiro del EPP ha sido adaptado al contexto de una cirugía ${ }^{[24,25]}$. El método de colocación paso a paso del EPP se describe en la Tabla 2.

Tabla 2. Secuencia de colocación del EPP, adaptado de las recomendaciones de la Asociación Española de Cirujanos y del documento técnico del Ministerio de Salud del Perú.

\section{Secuencia de colocación del EPP}

Retirar TODOS los objetos personales de los bolsillos

1 del pijama, así como cualquier accesorio antes de la colocación del EPP

2 Lavado de manos con agua y jabón o alcohol gel

3 Colocación de Scrub descartable

4 Colocar mascarilla N95 o superior

5 Colocar gorro, lentes protectores cerrados y botas

6 Colocar Overol

7 Colocación de mascarilla quirúrgica

8 Colocar segundo par de botas

9 Colocar pantalla facial

10 Remangar overol hasta el codo

11 Lavado de manos quirúrgico

12 Bajar manga por el personal de apoyo

13 Colocación de primer par de guantes cubriendo el overol

14 Entrada a quirófano, colocación de bata quirúrgica habitual (con ayuda para atarla)

15 Colocación de guantes estériles habituales. 


\section{SUGERENCIAS INTRAOPERATORIAS}

En el contexto de la pandemia por COVID-19, la mayoría de servicios opto por suspender todas las cirugías electivas, por lo que sólo se conservó la realización de cirugías de emergencia. En ese contexto, los servicios se enfrentan a tres posibles escenarios:

- Pacientes COVID-19 descartado.

- Pacientes con prueba rápida negativa, que no se puede descartar COVID-19.

- Pacientes COVID-19 sospechosos o confirmados.

Un paciente con prueba rápida negativa puede tener la enfermedad, si se encuentra en el periodo de incubación o si el resultado es falso negativo; por lo tanto, el manejo intraoperatorio en todos los casos será como si el paciente fuera confirmado a COVID-19. Sólo cabe el manejo intraoperatorio convencional, si al paciente se le ha descartado COVID-19 en el momento de la evaluación en emergencia, con una prueba molecular.

\section{Técnica quirúrgica}

La técnica empleada y protección del personal de salud se describen de acuerdo al escenario al que nos enfrentemos:

\section{Paciente COVID-19 descartado:}

- Técnica empleada según preferencia del cirujano

- Requiere uso de mascarilla N95 y lentes de montura integral o pantalla facial completa

Pacientes con prueba rápida negativa, que no se puede descartar COVID-19:

- Técnica empleada según preferencia del cirujano:

- Si se realiza cirugía laparoscópica, usar sistema de filtro de humo para eliminación de bacterias y virus (que filtren partículas a partir de $60 \mathrm{~nm}$ ), evitar fuga de $\mathrm{CO} 2$ durante la cirugía (se recomienda fijar los trócares a la pared abdominal o utilizar trócares con balón) y al eliminar el $\mathrm{CO} 2$, hacerlo con un sistema de aspiración cerrado.

- Si se realiza cirugía abierta se debe garantizar aspirar completamente el humo quirúrgico durante toda la cirugía.

- En todos los casos usar el EPP.

- La cantidad de personas dentro del quirófano debe ser lo menor posible.

- Debe operar el cirujano de mayor experiencia para minimizar el tiempo operatorio.

Paciente COVID-19 sospechoso o confirmado:

En un estudio de cohorte internacional publicado por COVIDSurg Collaborative ${ }^{[26]}$, se encontró que la mortalidad de pacientes operados con COVID-19 es del 23,8\%, las complicaciones pulmonares alcanzan el 51,2\%, y dentro de los pacientes con complicaciones pulmonares, la mortalidad es del $82,6 \%$.
Los predictores de mortalidad a los 30 días más importantes fueron: Edad mayor a 70 años, clasificación ASA III-V y presencia de comorbilidades. Destaca dentro de los resultados que la mortalidad en menores de 29 años fue del $0 \%$, pacientes sin comorbilidades y con ASA I-II, tuvieron un OR=1 (IC95\%), como predictores de mortalidad.

Por lo tanto, el manejo quirúrgico de los pacientes COVID-19, depende de la clasificación clínica (caso leve, moderado y severo) ${ }^{[16,27]}$ y de las comorbilidades asociadas:

Caso leve de COVID-19, sin comorbilidades: Manejo quirúrgico, abordaje según preferencia del cirujano.

Caso leve de COVID-19, con comorbilidades: Manejo médico de ser posible, sólo manejo quirúrgico si se justifica, a pesar del riesgo que implica la cirugía.

Caso moderado y severo de COVID-19, con o sin comorbilidades: Manejo médico de ser posible, sólo manejo quirúrgico si se justifica, a pesar del riesgo que implica la cirugía.

- Si se realiza cirugía laparoscópica utilizar filtros de humo quirúrgico.

- Si se realiza cirugía abierta, evitar usar electrocauterio, y de utilizarse se debe garantizar. aspirar completamente el humo durante toda la cirugía.

- En todos los casos usar el EPP.

- La cantidad de personas dentro del quirófano debe ser lo menor posible.

- Debe operar el cirujano de mayor experiencia para minimizar el tiempo operatorio.

La mejor forma de evitar dispersión del humo quirúrgico con aerosoles, es usando filtros de humo para bacterias/virus, con un sistema de aspiración cerrado para eliminar el $\mathrm{CO}_{2}$ al final de la cirugía, el cual puede hacerse con dispositivos comerciales o elaborarse un sistema de aspiración cerrado siguiendo recomendaciones internacionales, eso no sucede en cirugía abierta donde es difícil controlar la dispersión del humo quirúrgico ${ }^{[17,28,29]}$.

No hay consenso ni evidencia suficiente para recomendar hacer una ostomía o anastomosis primaria, la ostomía puede ser un foco de contaminación por la presencia del virus en heces ${ }^{[25]}$. Por otro lado, la anastomosis primaria laparoscópica puede recomendarse porque se evita el contacto del cirujano con la secreción del tubo digestivo. Sin embargo, la anastomosis abierta significa una exposición mayor y si se produce una fístula o una dehiscencia la contaminación será mayor, poniendo en riesgo de contagio a mayor cantidad de personal de salud. Al no haber consenso al respecto se deja a criterio del cirujano la decisión de una u otra técnica individualizando cada caso.

\section{SUGERENCIAS POSOPERATORIAS}

\section{Recuperación después de la cirugía}

El periodo de recuperación debe ser dado en la misma sala de operaciones, hasta que pase a una sala de aislamiento o UCl específica ${ }^{[30]}$ 


\section{Retiro del EPP (Tabla 3)}

Los médicos pueden ser más propensos a infectarse al retirarse que al colocarse el EPP, todos en sala de operaciones deben saber colocarse y retirárselo correctamente. La colocación y retiro del EPP debe ser revisado por el cirujano principal, residentes, anestesiólogos y enfermería antes de cada caso ${ }^{[17,31]}$.

Tabla 3. Secuencia de retiro del EPP, adaptado de las recomendaciones de la Asociación Española de Cirujanos y del documento técnico del Ministerio de Salud del Perú.

\section{Secuencia de retirada del EPP}

Retirarse la bata y guantes quirúrgicos como se

1 hace habitualmente, plegarla con la parte externa hacia dentro, y los guantes vueltos sobre si mismos. Desechar en el contenedor.

2 Lavado de guantes interiores con solución a base de alcohol

3 Retirar pantalla facial, agachar la cabeza, cerrando ojos y boca

4 Lavado de guantes interiores con solución a base de alcohol

5 Retirar mascarilla quirúrgica Lavado de guantes interiores con solución a base de alcohol

7 Retirar segundo par de botas

8 Lavado de guantes interiores con solución a base de alcohol

9 Retirar overol

10 Lavado de guantes interiores con solución a base de alcohol

11 Retirar lentes

12 Lavado de guantes interiores con solución a base de alcohol

13 Retiro de guantes interiores

14 Higiene quirúrgica de manos

15 Colocación de manoplas

16 Retiro de gorro

17 Lavado de manos con solución a base de alcohol

18 Retirar mascarilla N95 o superior

19 Lavado de manos con solución a base de alcohol

20 Retiro de botas

21 Lavado de manos con solución a base de alcohol

22 Retiro de scrub

23 Ducha

La antesala, que estaría inmediatamente al alcance de la sala de operaciones, sirve también como zona para el descarte del
EPP contaminado del personal de salud culminada la cirugía, así mismo debe estar libre de materiales no esenciales, alejadas de áreas de alto tráfico y debidamente señalada ${ }^{[31]}$. Si no es posible establecer una antesala, otro ambiente disponible exclusivamente para el manejo de material potencialmente contaminado puede ser suficiente ${ }^{[21]}$.

\section{Sistema de traslado del paciente y personal de salud}

- El traslado está cargo de dos personas, un médico/enfermero y un personal de apoyo, teniendo consideración en la protección personal, colocar el transportador plano de cama y colocar sábanas desechables. Después de la transferencia la sala y los equipos deben ser desinfectados con hipoclorito de sodio al $0,1 \%{ }^{[21]}$.

- La ruta de salida debe estar establecida según protocolos institucionales

- La enfermera circulante y el personal de apoyo externo trasladaran al paciente de regreso a sala de aislamiento.

- El personal de apoyo y el anestesiólogo trasladaran al paciente de regreso a $\mathrm{UCl}$ si es necesario. 30 minutos antes de salir se debe activar el sistema de seguridad de aviso de salida ${ }^{[20]}$

- La seguridad del hospital es responsable de despejar la ruta desde la sala o quirófano hacia la unidad de aislamiento, incluidos los ascensores ${ }^{[32]}$.

\section{Condiciones e indicaciones de alta de un paciente quirúrgico}

El alta debe estar cargo de un equipo multidisciplinario ante un paciente con sospecha o confirmación de infección por COVID-19. Serán las mismas que establece según normas institucionales, nacionales e internacionales:

- Cumplir los criterios quirúrgicos de alta según patológica especifica.

- La fiebre posoperatoria en cualquier paciente, es una causal para aplicar protocolos de descarte de infección por COVID-19 ${ }^{[21]}$

- Por lo general, no son necesarios medicamentos antivirales después del alta.

- Los pacientes deberán aislarse en sus domicilios por un periodo de dos semanas tras el alta. Se recomienda lo siguiente para el aislamiento:

- Aislarse en un ambiente totalmente independiente con ventilación adecuada y desinfección correspondiente.

- Evitar el contacto estricto con personas vulnerables como ancianos, niños o inmunodeprimidos.

- Uso frecuente de mascarillas y lavado de manos continuo, del paciente, así como los miembros de la familia.

- Medición de temperatura corporal mínimo dos veces al día y tener en cuenta los signos de alarma, así como el estado general del paciente. 


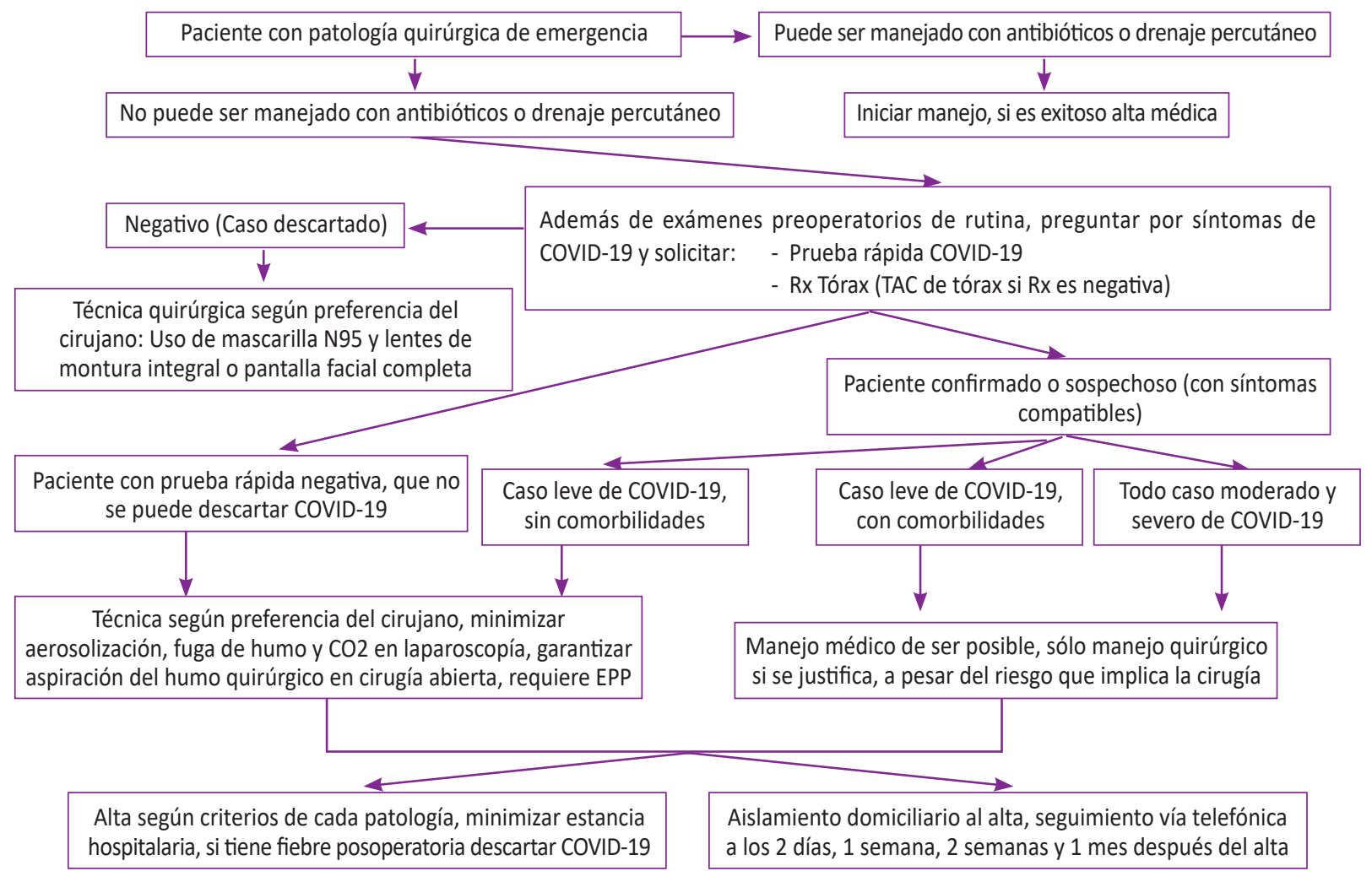

Figura 1. Flujograma propuesto del manejo quirúrgico de emergencia en cirugía general.

Un médico especialista debe asignar seguimientos individualizados para cada paciente. El primer control se debe llevar a cabo idealmente dentro de las 48 horas. Se continuará con el seguimiento después de una semana, dos semanas y un mes posterior al alta. El seguimiento será de manera virtual y se realizará a todos los pacientes, no solo a los confirmados o sospechosos de COVID-19, porque si el paciente tuvo prueba rápida negativa en el preoperatorio, es probable que haya estado en el período de incubación de la enfermedad, que sea un falso negativo o que se haya contagiado en el hospital. Ante cualquier eventualidad y/o complicación médica o quirúrgica debe acudir necesariamente al servicio de emergencia ${ }^{[16,24]}$.

\section{CONSIDERACIONES FINALES}

El escenario ideal sería contar con pruebas moleculares para el diagnóstico preoperatorio de COVID-19, sin embargo, en nuestro medio el resultado de una prueba molecular se obtiene en tres días; por lo tanto, no es aplicable en el contexto de una cirugía de emergencia donde se requiere el resultado de inmediato, esto contrasta con otras realidades donde el resultado de una prueba molecular se obtiene en cuatro horas ${ }^{[25]}$, es por este motivo que resulta más práctico utilizar pruebas rápidas en el preoperatorio.

La secuencia para colocarse y retirarse el EPP que recomiendan la OMS y el MINSA ${ }^{[24]}$, está enfocada a la atención de un paciente sospechoso de COVID-19, pero no se orientan al acto quirúrgico donde también se debe garantizar la correcta asepsia y antisepsia para prevenir infecciones intrahospitalarias. Por ejemplo, la OMS y el MINSA indican que luego del lavado de manos y el calzado de guantes se debe proceder a la colocación de la mascarilla y el gorro, lo que no es adecuado porque esos implementos no son estériles; por ello, la secuencia de colocación y retiro del EPP debe ser adaptada al contexto de una cirugía, lo que se sugiere en este artículo. Las recomendaciones vertidas se encuentran resumidas en una propuesta de flujograma de manejo (Figura 1).

Se concluye que, para el manejo quirúrgico de estos pacientes, es de suma importancia el uso adecuado de los equipos de protección personal, para evitar el contagio al personal de salud por la aerosolización del virus producida durante la cirugía; por ello, es necesario que el hospital provea de este material a los cirujanos. Además, se concluye que es factible realizar un abordaje abierto o laparoscópico en cirugías de emergencia con los cuidados adecuados tanto para el paciente como para el personal de salud; sin embargo, debemos considerar que las recomendaciones pueden cambiar con el tiempo al generarse nuevos conocimientos.

\section{ORCID:}

Yeray Trujillo-Loli, https://orcid.org/0000-0003-2829-5085

Alejandro Cabrera-Pastor, https://orcid.org/0000-0003-38225793 
Llenner Castañeda Puicón, https://orcid.org/0000-0003-4176$814 \mathrm{X}$

Contribución de autoría: YTL, ACP y LCP han participado en la concepción y diseño del artículo, su redacción y aprobación de la versión final a publicar.

Potenciales conflictos de interés: los autores declaran no tener conflictos de interés.

Fuente de financiamiento: autofinanciado.

\section{REFERENCIAS BIBLIOGRÁFICAS}

1. Organización Mundial de la Salud. Novel Coronavirus (2019-nCoV). Situation Report - 22. [Internet]. Ginebra: OMS; 2020 [citado 7 julio 2020]. Disponible en: https://www.who.int/docs/defaultsource/coronaviruse/situation-reports/20200211-sitrep-22-ncov. pdf?sfvrsn=fb6d49b1_2

2. Gobierno del Perú. Presidente Vizcarra dio a conocer primer caso de infección por coronavirus en el Perú e hizo un llamado a la población a mantener la calma [Internet]. Lima, Perú: Gobierno del Perú; 2020 [citado 5 abril 2020]. Disponible: https://www.gob. pe/institucion/presidencia/noticias/86976-presidente-vizcarra-dioa-conocer-primer-caso-de-infeccion-por-coronavirus-en-el-peru-ehizo-un-llamado-a-la-poblacion-a-mantener-la-calma

3. Bundu I, Patel A, Mansaray A, Kamara TB, Hunt LM. Surgery in the time of Ebola: how events impacted on a single surgical institution in Sierra Leone. J R Army Med Corps. 2016;162(3):212-6. doi: 10.1136/ jramc-2015-000582.

4. Red Nacional de Vigilancia Epidemiológica. Análisis de los casos de COVID-19 en personal sanitario notificados a la RENAVE hasta el 10 de mayo en España [Internet]. Madrid: Instituto de Salud Carlos III; 2020 [citado 10 julio 2020]. Disponible en: https://www. isciii.es/QueHacemos/Servicios/VigilanciaSaludPublicaRENAVE/ EnfermedadesTransmisibles/Documents/INFORMES/Informes\%20 COVID-19/COVID-19\%20en\%20personal\%20sanitario\%2029\%20 de\%20mayo\%20de\%202020.pdf

5. Wang D, Hu B, Hu C, Zhu F, Liu X, Zhang J, et al. Clinical Characteristics of 138 Hospitalized Patients With 2019 Novel Coronavirus-Infected Pneumonia in Wuhan, China. JAMA. American Medical Association; 2020;323(11):1061-9. doi: 10.1001/jama.2020.1585.

6. Álvarez Gallego M, Gortázar de las Casas S, Pascual Migueláñez I, Rubio-Pérez I, Barragán Serrano C, Álvarez Peña E, et al. Impacto de la pandemia por SARS-CoV-2 sobre la actividad y profesionales de un Servicio de Cirugía General y del Aparato Digestivo en un hospital terciario. Cir Esp. 2020. doi: 10.1016/j.ciresp.2020.04.001.

7. Wang W, Xu Y, Gao R, Lu R, Han K, Wu G, et al. Detection of SARS-CoV-2 in Different Types of Clinical Specimens. JAMA. 2020;323(18):1843-4. doi: 10.1001/jama.2020.3786.

8. Kwak HD, Kim S-H, Seo YS, Song K-J. Detecting hepatitis B virus in surgical smoke emitted during laparoscopic surgery. Occup Environ Med. 2016;73(12):857-63. doi: 10.1136/oemed-2016-103724.

9. van Doremalen N, Bushmaker T, Morris DH, Holbrook MG, Gamble A, Williamson BN, et al. Aerosol and Surface Stability of SARS-CoV-2 as Compared with SARS-CoV-1. N Engl J Med. 2020. doi: 10.1056/ NEJMc2004973.

10. Okoshi K, Kobayashi K, Kinoshita K, Tomizawa Y, Hasegawa S, Sakai Y. Health risks associated with exposure to surgical smoke for surgeons and operation room personnel. Surg Today. 2015;45(8):957-65. doi: 10.1007/s00595-014-1085-z.
11. Coia JE, Ritchie L, Adisesh A, Booth CM, Bradley C, Bunyan D, et al. Guidance on the use of respiratory and facial protection equipment. J Hosp Infect. 2013;85(3):170-82. doi: 10.1016/j.jhin.2013.06.020.

12. Maclntyre CR, Seale H, Dung TC, Hien NT, Nga PT, Chughtai AA, et al. A cluster randomised trial of cloth masks compared with medical masks in healthcare workers. BMJ Open. 2015;5(4):e006577. doi: 10.1136/bmjopen-2014-006577.

13. Segura Sanpedro J, Reyes ML, García-Granero Á, De la Portilla F. Recomendaciones de actuación patología colorrectal de la AECP ante COVID-19 [Internet]. Documento 1_V1_marzo 2020. Madrid: Asociación Española de Coloproctología; 2020 [citado 10 julio 2020]. Disponible en: https://aecp-es.org/images/site/covid/ DOCUMENTO_1.pdf

14. World Health Organization. Coronavirus disease 2019 (COVID-19). Situation report - 155 [Intenet]. Geneva: WHO; 2020. Disponible en: https://www.who.int/docs/default-source/coronaviruse/situationreports/20200623-covid-19-sitrep-155.pdf?sfvrsn=ca01ebe_2

15. National Guideline Centre. Preoperative Tests (Update): Routine Preoperative Tests for Elective Surgery [Intenet]. London: National Institute for Health and Care Excellence (UK); 2016 [citado 10 julio 2020]. Disponible en: http://www.ncbi.nlm.nih.gov/books/ NBK355755/

16. Liang T, Cai H, Chen Y, Chen Z, Fang $Q$, Han W, et al. Handbook of COVID-19 Prevention and Treatment [Internet]. Zhejiang: Universidad de Zhejiang; 2020 [citado 10 julio 2020]. Disponible en: https://esge.org/documents/Handbook_of_COVID-19_ Prevention_and_Treatment.pdf

17. Asociación Española de Cirujanos. Recomendaciones para manejo de pacientes con infección por COVID-19 en el contexto de una intervención quirúrgica urgente o electiva [Internet]. Madrid: AEC; 2020 [citado 10 julio 2020]. Disponible en: https://www. aecirujanos.es/files/noticias/152/documentos/Recomendaciones_ caso_cirugia.pdf

18. American College of Surgeons. American College of Surgeons. 2020. COVID-19: Elective Case Triage Guidelines for Surgical Care [Internet]. Chicago, IL: ACS; 2020 [citado 10 julio 2020]. Disponible en: https://www.facs.org/covid-19/clinical-guidance/elective-case

19. Royal College of Surgeons of England. Updated Intercollegiate General Surgery Guidance on COVID-19 [Internet]. London: RCS; 2020 [citado 10 julio 2020]. Disponible en: https://www.rcseng. ac.uk/coronavirus/joint-guidance-for-surgeons-v2/

20. Wax RS, Christian MD. Practical recommendations for critical care and anesthesiology teams caring for novel coronavirus (2019-nCoV) patients. Can J Anesth. 2020;67(5):568-76. doi: 10.1007/s12630020-01591-x.

21. Tao KX, Zhang BX, Zhang P, Zhu P, Wang GB, Chen XP, et al. [Recommendations for general surgery clinical practice in novel coronavirus pneumonia situation]. Zhonghua Wai Ke Za Zhi. 2020;58:E001. doi: 10.3760/cma.j.issn.0529-5815.2020.0001.

22. Trujillo CHS. Consenso colombiano de atención, diagnóstico y manejo de la infección por SARS-COV-2/COVID 19 en establecimientos de atención de la salud. Recomendaciones basadas en consenso de expertos e informadas en la evidencia. Infectio. 2020;24(3):1-153. doi: 10.22354/in.v24i3.851.

23. Instituto de Evaluación de Tecnologías en Salud e Investigación. Recomendaciones para realizar procedimientos quirúrgicos en el marco de la pandemia por COVID-19 [Internet]. Lima: EsSalud; 2020 [citado 10 julio 2020]. Disponible en: http://www.essalud.gob.pe/ ietsi/pdfs/guias/Recomendaciones_procedimientos_quirurgicos_ COVID19.pdf

24. Ministerio de Salud del Perú. Documento Técnico. Atención y manejo clínico de casos de COVID-19. Escenario de transmisión focalizada [Internet]. Lima, Perú: MINSA; 2020 [citado 10 
julio 2020]. Disponible en: https://cdn.www.gob.pe/uploads/ document/file/545934/07_03_FINAL_BCM_DOCUMENTO_ TE\%CC\%81CNICO_002_.pdf

25. Balibrea JMose, Badia JMose, Rubio Pérez I, Martín Antona E, Álvarez Peña E, García Botella S, et al. Manejo quirúrgico de pacientes con infección por COVID-19. Recomendaciones de la Asociación Española de Cirujanos. Cir Espan. 2020;98(5):251-9. doi: 10.1016/j.ciresp.2020.03.001.

26. Nepogodiev D, Bhangu A, Glasbey JC, Li E, Omar OM, Simoes JF, et al. Mortality and pulmonary complications in patients undergoing surgery with perioperative SARS-CoV-2 infection: an international cohort study. Lancet. 2020;396(10243):27-38. doi: 10.1016/\$01406736(20)31182-X.

27. Mendoza-Ticona A, Mesias GV, Aquehua AQ, Chacaliaza BC, Loli GG, Cruz CA, et al. Clasificación clínica y tratamiento temprano de la COVID-19. Reporte de casos del Hospital de Emergencias Villa El Salvador, Lima-Perú. Act Med Peru. 2020;37(2). doi: 10.35663/ amp.2020.372.968.

28. Society of American Gastrointestinal and Endoscopic Surgeons. Resources for smoke \& gas evacuation during open, laparoscopic and endoscopic procedures [Internet]. Los Angeles: CASAGES; 2020 [citado 10 julio 2020]. Disponible: https://www.sages.org/ resources-smoke-gas-evacuation-during-open-laparoscopicendoscopic-procedures/

29. Mintz Y, Arezzo A, Boni L, Chand M, Brodie R, Fingerhut A, et al. A Low-cost, Safe, and Effective Method for Smoke Evacuation in Laparoscopic Surgery for Suspected Coronavirus Patients. Ann Surg. 2020;272(1):e7-8. doi: 10.1097/SLA.0000000000003965.

30. Ti LK, Ang LS, Foong TW, Ng BSW. What we do when a COVID-19 patient needs an operation: operating room preparation and guidance. Can J Anesth. 2020;67(6):756-8. DOI: 10.1007/s12630020-01617-4.

31. Elizabeth Brindle M, Gawande A. Managing COVID-19 in Surgical Systems. Ann Surg. 2020;272(1):e1-2. doi: 10.1097/ SLA.0000000000003923.

32. Brat GA, Hersey S, Chhabra K, Gupta A, Scott J. Protecting Surgical Teams During the COVID-19 Outbreak: A Narrative Review and Clinical Considerations. Ann Surg. 2020. doi: 10.1097/ SLA.0000000000003926. 